\title{
The impact of induction therapy with three doses of infliximab on deep histological healing in paediatric patients with active Crohn's disease
}

\author{
Edyta Szymanska1, Maciej Dadalski², Sylwia Szymanska³, Wieslawa Grajkowska³, Maciej Pronicki \\ Jaroslaw Kierkus ${ }^{2}$ \\ ${ }^{1}$ Department of Paediatrics, Nutrition, and Metabolic Disorders, Children's Memorial Health Institute, Warsaw, Poland \\ 2Department of Gastroenterology, Hepatology, and Feeding Disorders, Children's Memorial Health Institute, Warsaw, Poland \\ ${ }^{3}$ Department of Pathology, Children's Memorial Health Institute, Warsaw, Poland
}

Gastroenterology Rev 2016; 11 (3): 176-180 DOI: $10.5114 / p g .2016 .57753$

Key words: deep remission, histological healing, mucosal healing, biological therapy, Crohn's disease.

Address for correspondence: Edyta Szymanska MD, Department of Paediatrics, Nutrition, and Metabolic Disorders, Children's Memorial Health Institute, Al. Dzieci Polskich 20, 04-730 Warsaw, Poland, phone: +48 513017 570, e-mail: edyta.szymanska@onet.com.pl

\begin{abstract}
Introduction: The clinical efficacy of infliximab (IFX) for induction of remission in both adults and children with active Crohn's disease (CD) has been well documented. Recently, so-called "deep remission" defined as mucosal healing has become the ultimate endpoint of the most recent therapeutic advances for CD. However, endoscopic evidence of mucosal healing is not necessarily associated with histological evidence of suppression of inflammation.

Aim: Since data on that issue are limited, especially in the paediatric population, the aim of this study was to assess the impact of induction therapy with IFX on deep microscopic remission in paediatric patients with CD.

Material and methods: Fifty-six children (32 boys and 24 girls) aged $13.0 \pm 9.3$ years with moderate to severely active CD diagnosed at the mean age of $5.5 \pm 0.83$ years were included into the study. Colonoscopy and gastroscopy with sample collection were performed in all patients before and after three injections of IFX. Clinical activity of the disease was assessed using the Paediatric Crohn's Disease Activity Index (PCDAI), and the endoscopic activity was scored using the Simple Endoscopic Score (SES-CD). Histological changes were evaluated by a previously described numerical scoring system.

Results: Thirty-nine (69.6\%) patients reached clinical remission (PCDAI $\leq 10)$. When comparing data at baseline and at week 10 , a significant decrease was observed in median PCDAI, and in SES-CD score between the initial and control colonoscopies. We also reported a decrease in histological scale. However, the difference was not statistically significant $(p=0.63)$. Three (5.4\%) patients had a score of zero in the control histological examination. The correlation was found only between histological score and SES-CD score. Clinical remission correlated better with mucosal healing expressed by a decrease in SES-CD score than with microscopic changes.

Conclusions: Biological therapy with infliximab enables mucosal healing in paediatric patients with $C D$, which is not necessarily associated with histological evidence of suppression of inflammation. Mucosal healing correlates better than microscopic healing with clinical remission.
\end{abstract}

\section{Introduction}

Crohn's disease (CD) is an inflammatory condition of unknown etiology, which is classified in the inflammatory bowel disease (IBD) group along with ulcerative colitis (UC) [1, 2]. Since the pathogenesis of this condition has not been established yet, there is no causal treatment for CD [3]. Consequently, the goals of both the pharmacotherapy and surgical management are limited to obtaining the longest possible remission and preventing a relapse $[4,5]$. However, in recent years a novel biologic therapy with monoclonal antibodies against tumor necrosis factor (TNF)- $\alpha$ has revolutionised the treatment of IBD [6, 7]. Biologic agents such as infliximab (IFX) and adalimumab (ADA) have been demonstrated to improve to a considerable degree both the clinical and endoscopic variables [8-10]. Therefore, the "ideal" treatment of CD should not only suppress symptoms, but if at all possible, additionally heal mu- 
cosal lesions [11]. Since mucosal healing $(\mathrm{MH})$ might be the only way to alter the disease course in IBD patients, new guidelines recommend that it should be the ultimate endpoint in clinical trials [12]. In CD deep remission has recently been defined as Crohn's Disease Activity Index $(\mathrm{CDAl})<150$ and complete $\mathrm{MH}$ [13]. However, in the near future the concept of deep remission might include microscopic-histological healing in CD. It has been suggested that 'deep' remission, i.e. beyond the control of clinical symptoms, might ultimately have an impact on important long-term outcomes such as the need for surgery and the development of disability $[14,15]$. However, there have been studies demonstrating little correlation between endoscopic $\mathrm{MH}$ and clinical remission of the disease, as determined by the Crohn's disease activity index (PCDAI) $[16,17]$. Moreover, endoscopic evidence of mucosal healing was not necessarily associated with histological evidence of suppression of inflammation [18, 19]. Nonetheless, it must be stressed that in CD histological assessment of mucosal healing is the only way to establish in an objective manner the reversion of the mucosa to a normal state [20]. Nonetheless, deep histological remission is at present not recommended as a primary endpoint for therapeutic trials because of the lack of a standardised approach.

\section{Aim}

Therefore, since there are no data on deep histological remission in children with CD, the aim of this study was to verify the impact of induction therapy with IFX on mucosal healing with particular consideration paid to microscopic improvement.

\section{Material and methods}

A retrospective analysis of patients diagnosed with $C D$ and treated with biologic therapy with IFX at the Department of Gastroenterology, Hepatology, and Feeding Disorders, Children's Memorial Health Institute was performed. The analysis included an 8-year period of time - between 2005 and 2013. Detailed characteristics of the analysed patients are presented in Table I.

The primary objective of this study was to evaluate the clinical, endoscopic, and microscopic activity of the disease in our patients prior to treatment (baseline) and after induction therapy (endpoint) with three doses of IFX. Disease activity was evaluated using PCDAI, which takes into account both clinical and biochemical parameters. Clinical remission was defined as PCDAI < 10 . Selected endoscopic parameters (ulcer size, ulcerated and affected surfaces, stenosis) were scored from zero to three using the Simple Endoscopic Scale for Crohn's Disease (SES-CD). Mucosal healing was defined as a complete absence of ulcers or a significant decrease in SES-CD as a sign of inflammatory lesion decrease in the presence of ulcerated surfaces, while stationary endoscopic features stood for the lack of progression or regression. Histological changes were precisely described and evaluated using a previously described numerical scoring system for CD, which was adopted for the purpose of this study (Table II). Deep microscopic remission was defined as a complete absence of inflammation in histological examination or a significant decrease in the scoring of the histological scale. Stationary microscopic features were considered as the lack of progression or regression.

\section{Statistical analysis}

The proportion of patients in clinical response and in clinical remission at week 10 was assessed. All analyses were based on a per-protocol principle. The Wilcoxon test was used to compare quantitative variables, and $p<0.05$ was regarded as significant. Scorings in SES-CD and histological grading scale were used as two independent variables in the analysis of discrimination between the group with clinical response and the group with no response.

Table I. Detailed characteristics of CD patients treated with biologic therapy $(n=56)$

\begin{tabular}{lc}
\hline Parameter & Characteristic \\
\hline Gender: & $32(57.1 \%)$ \\
\hline Males & $24(42.9 \%)$ \\
\hline Females & $13.0 \pm 6.3$ \\
\hline Age during biologic therapy [years] & $18.5 \pm 8.5$ \\
\hline Mean duration time of disease [months] & $19.6 \%$ \\
\hline PCDAl, median (interquartile range) & $21.4 \%$ \\
\hline Involved region: & $48.2 \%$ \\
\hline Isolated ileal disease (L1) & $10.8 \%$ \\
\hline Colonic disease (L2) & $10.0(0.0-32.0)$ \\
\hline Ileocolon (L3) & $5.5(0.0-10.0)$ \\
\hline Upper disease (L4) & \\
\hline SES-CD, median (interquartile range) & $5(12.5 \%)$ \\
\hline Histological score, median (interquartile range) & $56(100 \%)$ \\
\hline Previous treatment: & $56(100 \%)$ \\
\hline AZA & \\
\hline GKS & \\
\hline MTX & \\
\hline Cyclosporine & \\
\hline AZA & \\
\hline
\end{tabular}

AZA - azathioprine, GKS - steroid, MTX - methotrexate. 
Table II. Self-adapted numerical histologic scoring system for CD based on a score of 0-3 of the following five segments of intestine: ileum, right colon, transverse colon, left colon, and rectum

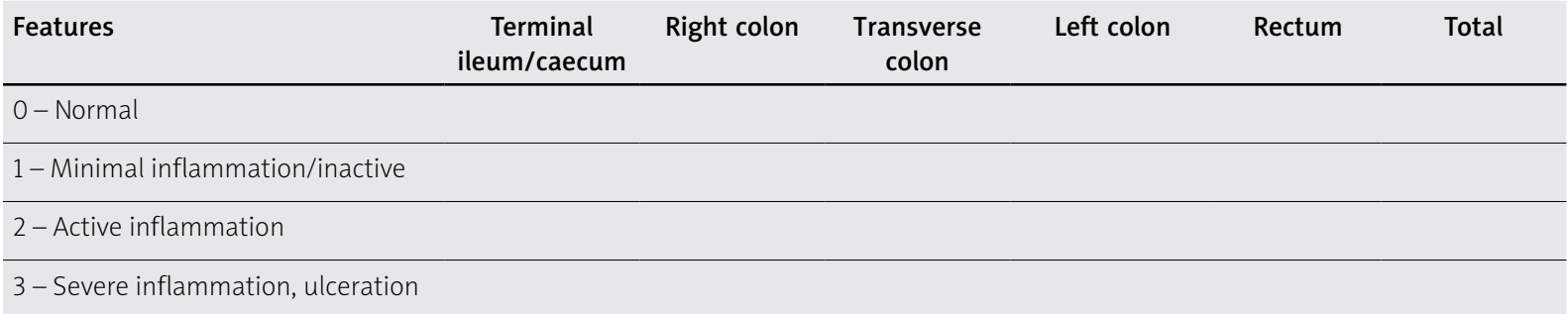

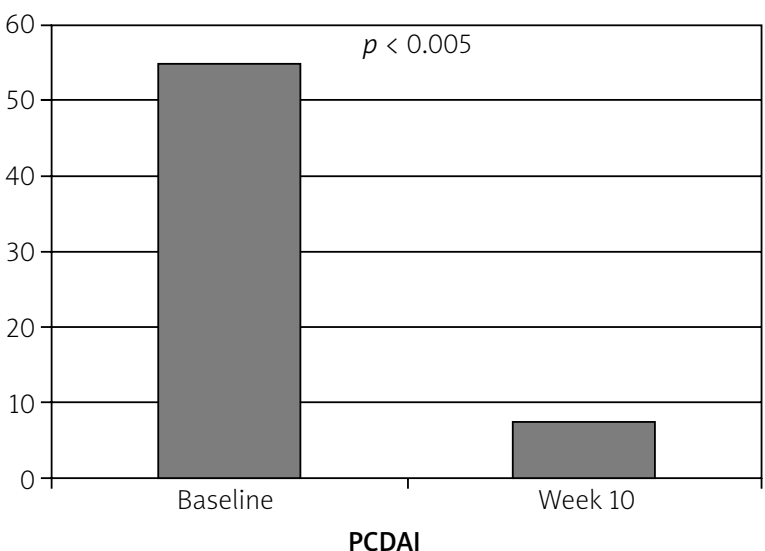

Figure 1. Clinical efficacy of induction therapy with three doses of IFX as determined by a significant decrease in PCDAI between baseline (week 0) and endpoint (week 10)

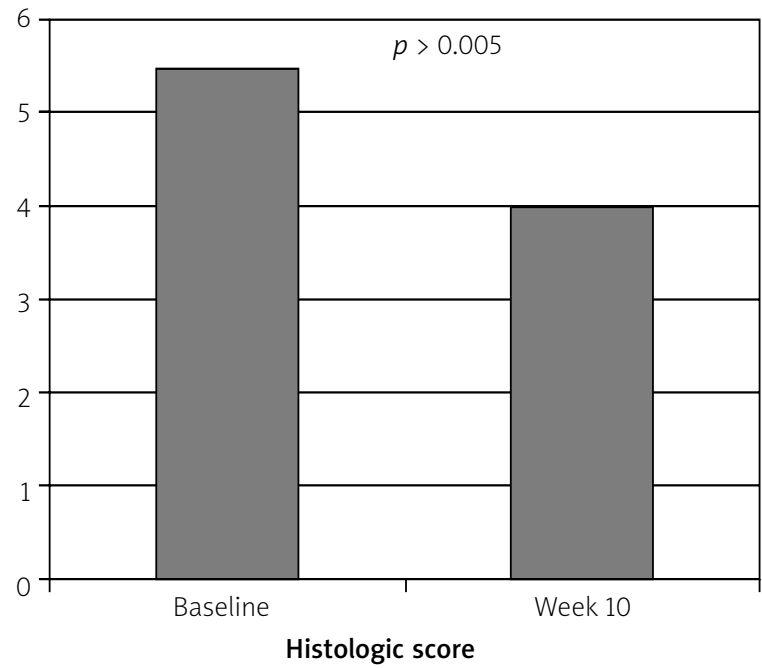

Figure 3. Impact of induction therapy on histological-intestinal healing with three doses of IFX as determined by a decrease in the previously described numerical scoring system between the initial (week 0) and the control (week 10) colonoscopy - decrease not significant statistically $(p=0.63)$

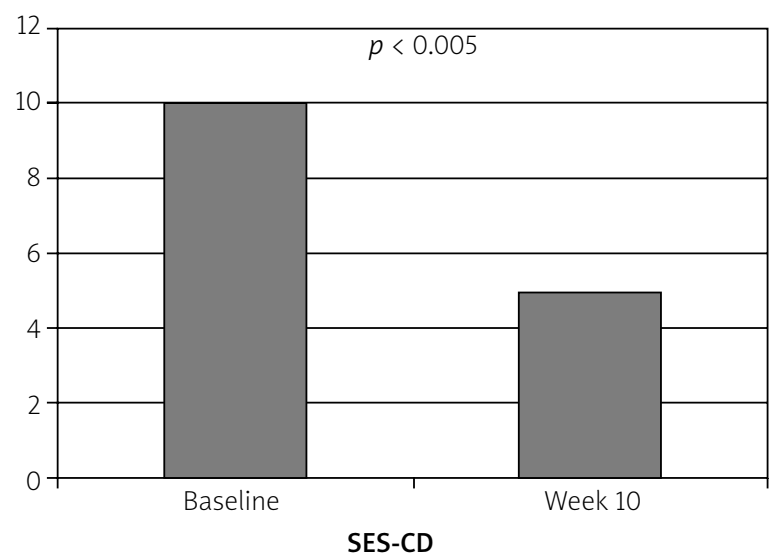

Figure 2. Impact on mucosal healing by induction therapy with three doses of IFX as determined by a significant decrease in SES-CD between the initial (week 0) and the control (week 10) colonoscopy

\section{Results}

Thirty-nine (69.6\%) patients reached clinical remission; 17 (30.4\%) children either did not respond to the therapy $(11.8 \%)$ or did not enter full remission (88.2\%). When comparing data at baseline and at week 10 , a significant decrease was observed in median PCDAI (baseline median (interquartile range) 55 (40.0$85.0)$ vs. 7.5 (2.5-40.0) at week 10) (Figure 1), and in SES-CD score between the initial and control colonoscopies (10.0 (0.0-32.0) vs. $5.0(0.0-23.0)$ ) (Figure 2). Nine out of 56 patients had a score of 0 in control endoscopy vs. 3 out of 56 in initial colonoscopy. A decrease in histological score was reported (5.5 (0.0-10.0) vs. 4.0 (0.0-12.0)). However, the difference was not statistically significant $(p=0.63)$ (Figure 3$)$. Three (5.4.\%) patients had a score of 0 in the control histological examination.

Only SES-CD score had a significant impact on discrimination between the group with clinical remission vs. no remission, while histological changes did not discriminate significantly between those two groups. A correlation was found between histological score and SES-CD score. 


\section{Discussion}

The aim of this study was to provide evidence of a successful induction therapy with IFX as regards mucosal healing and deep remission expressed by histological-intestinal healing in children with moderate to severe CD. Current data on the mucosal effects of IFX are limited in the case of paediatric CD. This study was the first one to assess the impact of any (not only biologic) therapy on deep histological remission in children with CD. A few studies have been carried out on biological agents, but so far the only parameters by which treatment efficacy was assessed have been clinical remission and $\mathrm{MH}[8,9,21]$. However, endoscopic remission differs from histological remission [22], which unfortunately is difficult to assess in CD because inflammation in this type of IBD tends to be patchy, resulting in the need for multiple biopsies for evaluation. Moreover, a universally accepted histological system for grading inflammation in CD is lacking. Therefore, we decided to develop a self-adapted numerical scoring system for $C D$, based on the score of 0-3 of the following five segments of intestine: terminal ileum and caecum, right colon, transverse colon, left colon, and rectum. We are aware of certain limitations of that method. First of all, the scale has not been validated, and secondly, a patchy pattern of inflammation in CD makes microscopic evaluation difficult. Nonetheless, we assumed that a numerical system would be clearer and would better reflect histological changes than a traditional descriptive protocol. Infliximab has been proven to induce and maintain $\mathrm{MH}$ in the ACCENT 1 trial [23]. In our previous studies, we also demonstrated that biological therapy with IFX enabled mucosal healing in paediatric patients with CD [8]. Currently, however, the concept of deep remission might include also microscopic-histological healing in IBD. Crohn's disease, contrary to UC, is a transmural disease, and mucosal healing may not be sufficient even though the mucosa may return to almost normal after treatment. Deeply situated lesions may persist, and endoscopy with biopsy may not assess the deeper layers of the intestine [24]. That is why endoscopic evidence of mucosal healing is not necessarily associated with histological evidence of suppressed inflammation. Data on deep remission in CD are sparse. D'Haens et al. investigated histological healing in $30 \mathrm{CD}$ patients treated with IFX in a placebo-controlled trial. After induction therapy with three doses of either 5 , 10 , or $20 \mathrm{mg} / \mathrm{kg}$ of IFX or placebo as a single infusion, inflammatory infiltrate disappeared only in IFX-treated patients, but architectural changes persisted in most of them [25]. Another study by the same group evaluated the effects of a single infusion of IFX in 15 patients with steroid-refractory $\mathrm{CD}$. Histological activity scores, assessed at 4 weeks after infusion, showed (in comparison to placebo) a significant decrease mainly due to the reduction of inflammatory activity [26]. In our study, biological therapy with three induction doses of IFX resulted in macroscopic but not necessarily microscopic remission; the decrease in histological score was statistically insignificant ( $p=0.63$ ). Moreover, $\mathrm{MH}$ correlated better than microscopic healing with clinical remission. Taking into consideration that, as reported above, true histological healing may be obtained in only about $30 \%$ of treated patients, and that our paediatric patients presented mostly with severely active CD, better outcomes should rather not be expected. We are entirely aware of certain limitations of our study, such as retrospective analysis. However, the main value of our study assessing the impact of induction therapy with IFX on deep histological remission in children pertains to its novelty. It is the first such large study conducted on a paediatric population. The analysed cohort is greater even than the group of adults analysed so far by D'Haens. Additionally, the number of clinical trials and original papers dealing with deep remission in CD is limited. Furthermore, they did not focus on children. Such studies are difficult to perform because of the lack of a standardised approach. Therefore, additional studies searching for optimal sampling, standardising scores, and features should be performed.

\section{Conclusions}

According to our results, biological therapy with IFX is effective to achieve $\mathrm{MH}$ but not deep histological remission in paediatric patients with moderate to severely active CD. Mucosal healing correlated better than microscopic healing with clinical remission. Therefore, it seems that macroscopic remission expressed by $\mathrm{MH}$ together with clinical remission should remain the main endpoints of therapy because histological healing is difficult to assess due to the lack of optimal sampling and standardising scores.

\section{Conflict of interest}

The authors declare no conflict of interest.

\section{References}

1. Baumgart DC, Sandborn WJ. Inflammatory bowel disease: clinical aspects and established and evolving therapies. Lancet 2007; 369: 1641-57.

2. Hanauer SB. Inflammatory bowel disease. N Engl J Med 1996; 334: 841-8.

3. Benchimol El, Fortinsky KJ, Gozdyra P, et al. Epidemiology of pediatric inflammatory bowel disease: a systematic review of international trends. Inflamm Bowel Dis 2011; 17: 423-39.

4. Dignass A, Van Assche G, Lindsay JO, et al. The second European evidence-based consensus on the diagnosis and man- 
agement of Crohn's disease: Current management. J Crohns Colitis 2010; 4: 28-62.

5. Tung J, Loftus EV Jr, Freese DK, et al. A population-based study of the frequency of corticosteroid resistance and dependence in pediatric patients with Crohn's disease and ulcerative colitis. Inflamm Bowel Dis 2006; 12: 1093-100.

6. Cucchiara S, Morley-Fletcher A. "New drugs: kids come first": children should be included in trials of new biological treatments. Inflamm Bowel Dis 2007; 13: 1165-9; discussion 1176-7.

7. Rosenbach Y, Hartman C, Shapiro R, et al. Adalimumab treatment in children with refractory Crohn's disease. Dig Dis Sci 2010; 55: 747-53.

8. Kierkus J, Dadalski M, Szymanska E, et al. The impact of infliximab induction therapy on mucosal healing and clinical remission in Polish pediatric patients with moderate-to-severe Crohn's disease. Eur J Gastroenterol Hepatol 2012; 24 495-500.

9. D'haens G, Van Deventer S, Van Hogezand R, et al. Endoscopic and histological healing with infliximab anti-tumor necrosis factor antibodies in Crohn's disease: a European multicenter trial. Gastroenterology 1999; 116: 1029-34.

10. Paul S, Del Tedesco E, Marotte $\mathrm{H}$, et al. Therapeutic drug monitoring of infliximab and mucosal healing in inflammatory bowel disease: a prospective study. Inflamm Bowel Dis 2013 19: 2568-76.

11. D'Haens G. Mucosal healing in pediatric Crohn's disease: the goal of medical treatment. Inflamm Bowel Dis 2004; 10: 479-80.

12. Van Assche G, Dignass A, Panes J, et al.; the European Crohn's and Colitis Organisation (ECCO). The second European evidence-based consensus on the diagnosis and management of Crohn's disease: definitions and diagnosis. J Crohn's Colitis 2010; 4: 7-27.

13. Zallot C, Peyrin-Biroulet L. Deep remission in inflammatory bowel disease: looking beyond symptoms. Curr Gastroentero Rep 2013; 15: 315.

14. Ben-Horin S, Kopylov U, Chowers Y. Optimizing anti-TNF treatments in inflammatory bowel disease. Autoimmun Rev 2014 13: 24-30.

15. Colombel JF, Louis E, Peyrin-Biroulet L, et al. Deep remission: a new concept? Dig Dis 2012; 30: 107-11.

16. Munkholm P, Langholz E, Davidsen $M$, et al. Disease activity courses in a regional cohort of Crohn's disease patients. Scand J Gastroenterol 1995; 30: 699-706.

17. Pimentel-Nunes P, Dinis-Ribeiro M, Magro F. Systematic review on drug and diet-induced endoscopic remission in Crohn's disease. Eur J Gastroenterol Hepatol 2009; 21: 491-503.

18. Korelitz BI, Sommers SC. Response to drug therapy in Crohn's disease: evaluation by rectal biopsy and mucosal cell counts. J Clin Gastroenterol 1984; 6: 123-7.

19. D'Haens G, Geboes K, Rutgeerts P. Endoscopic and histologic healing of Crohn's (ileo-)colitis with azathioprine. Gastrointest Endosc 1999; 50: 667-71.

20. Kornbluth A, Sachar DB. Ulcerative colitis practice guidelines in adults (update): American College of Gastroenterology, Practice Parameters Committee. Am J Gastroenterol 2004; 99 : 1371-85

21. Hyams J, Crandall W, Kugathasan S, et al. Induction and maintenance infliximab therapy for the treatment of moder- ate-to-severe Crohn's disease in children. Gastroenterology 2007; 132: 863-73.

22. Sandborn WJ, Feagan BG, Hanauer SB, et al. A review of activity indices and efficacy endpoints for clinical trials of medical therapy in adults with Crohn's disease. Gastroenterology 2002; 122: 512-30

23. Hanauer SB, Feagan BG, Lichtenstein GR, et al. ACCENT I Study Group. Maintenance infliximab for Crohn's disease: the ACCENT I randomised trial. Lancet 2002; 359: 1541-9.

24. Frizelle FA, Ing A, Gearry RB, et al. Immunomodulation does not alter histology in resected Crohn's disease. Tech Coloproctol 2009; 13: 295-300.

25. D'Haens G, Van Deventer S, Van Hogezand R, et al. Endoscopic and histological healing with infliximab anti-tumor necrosis factor antibodies in Crohn's disease: a European multicenter trial. Gastroenterology 1999; 116: 1029-34.

26. Baert FJ, D'Haens GR, Peeters M, et al. Tumor necrosis factor alpha antibody (infliximab) therapy profoundly down-regulates the inflammation in Crohn's ileocolitis. Gastroenterology 1999; 116: 22-8.

Received: 9.03.2015

Accepted: 7.05.2015 